\title{
Evidence of nuclear disks in starburst galaxies from their radial distribution of supernovae ${ }^{\star}$
}

\author{
R. Herrero-Illana, M. Á. Pérez-Torres, and A. Alberdi \\ Instituto de Astrofísica de Andalucía - CSIC, PO Box 3004, 18008 Granada, Spain \\ e-mail: herrero@iaa.es
}

Received 29 November 2011 / Accepted 12 March 2012

\begin{abstract}
Galaxy-galaxy interactions are expected to be responsible for triggering massive star formation and possibly accretion onto a supermassive black hole, by providing large amounts of dense molecular gas down to the central kiloparsec region. Several scenarios to drive the gas further down to the central $\sim 100 \mathrm{pc}$, have been proposed, including the formation of a nuclear disk around the black hole, where massive stars would produce supernovae. Here, we probe the radial distribution of supernovae and supernova remnants in the nuclear regions of the starburst galaxies M 82, Arp 299-A, and Arp 220, by using high-angular resolution $\left(\$ 00^{\prime} 1\right)$ radio observations published in the literature (for M 82 and Arp 220), or obtained by ourselves from the European VLBI Network (Arp 299-A). Our main goal was to characterize the nuclear starbursts in those galaxies and thus test scenarios that propose that nuclear disks of sizes $\sim 100 \mathrm{pc}$ form in the central regions of starburst galaxies. We obtained the radial distribution of supernovae $(\mathrm{SNe})$ in the nuclear starbursts of M 82, Arp 299-A, and Arp 220, and derived scale-length values for the putative nuclear disks powering the bursts in those central regions. The scale lengths for the (exponential) disks range from $\sim 20-30 \mathrm{pc}$ for Arp 299-A and Arp 220, up to $140 \mathrm{pc}$ for M 82 . The radial distribution of SNe for the nuclear disks in Arp 299-A and Arp 220 is also consistent with a power-law surface density profile of exponent $\gamma=1$, as expected from detailed hydrodynamical simulations of nuclear disks. Our results support scenarios where a nuclear disk of size $\sim 100 \mathrm{pc}$ is formed in (U)LIRGs, and sustained by gas pressure, in which case the accretion onto the black hole could be lowered by supernova feedback.
\end{abstract}

Key words. galaxies: starburst - galaxies: luminosity function, mass function - galaxies: individual: Arp 299-A supernovae: general - radiation mechanisms: non-thermal - radio continuum: stars

\section{Introduction}

The activity in the central regions of luminous and ultraluminous infrared galaxies (LIRGs, $10^{11} L_{\odot} \leq L_{\mathrm{IR}}<10^{12} L_{\odot}$, and ULIRGs, $L_{\mathrm{IR}} \geq 10^{12} L_{\odot}$, respectively) is powered by either accretion onto a supermassive black hole (SMBH), and/or by a burst of activity due to young, massive stars. It has been found that LIRGs and ULIRGs are associated with interacting and merging galaxies, respectively, and the interaction between the galaxies is assumed to provide large amounts of dense molecular gas that eventually reach the central regions of the galaxies, where they are responsible for triggering massive star formation and possibly accretion onto an SMBH (e.g., Di Matteo et al. 2007). While interactions and bars seem to aid the transport of the molecular gas into the kiloparsec region of galaxies (e.g., Simkin et al. 1980), it has been recognized that a major difficulty is in driving the gas from the kiloparsec region down to the central parsec, or parsecs, of (U)LIRGs, where a nuclear starburst and/or an active galactic nucleus (AGN) are expected to exist. Several mechanisms have been proposed to overcome this problem, including tidal torques driven by either stellar bars (Shlosman et al. 1990) or major and minor mergers (Mihos \& Hernquist 1994, 1996), gas drag and dynamical friction in a dense nuclear star cluster (Norman \& Scoville 1988), or radiation drag (Umemura 2001; Kawakatu \& Umemura 2002). However, not all the accumulated gas is expected to be accreted

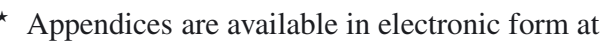
http://www . aanda.org
}

directly onto the putative SMBH, since the removal of the angular momentum of the gas is very inefficient (Umemura 2001). The angular momentum that is not removed will then permit the accreted gas to form a reservoir, namely a nuclear disk in the central $\sim 100$ pc around the AGN (e.g., Wada \& Norman 2002; Kawakatu \& Wada 2008, and references therein). Furthermore, if the gas in the nuclear starburst is dense enough, vigorous star formation is expected to occur, which can be detected via, e.g., high-angular resolution observations of recently exploded supernovae (e.g., in the LIRG Arp 299, Pérez-Torres et al. 2009; or the ULIRG Arp 220, Lonsdale et al. 2006). The nuclear starburst is also expected to affect the growth of the SMBH, since the radiation and/or supernova ( $\mathrm{SN}$ ) feedback caused by starbursts can eventually trigger the mass accretion onto a SMBH (e.g., Umemura et al. 1997; Wada \& Norman 2002).

Kawakatu \& Wada (2008) proposed an scenario for (U)LIRGs, where a nuclear disk is supported by the turbulent pressure of SNe. In their model, the turbulence due to supernova activity transports the angular momentum toward the central pcscale region. One of the implications of the model developed by Kawakatu \& Wada (2008) is that the BH growth rate depends on the spatial distribution of both young stars and SNe in the nuclear disk. However, the study of this spatial distribution is a challenging observational task for two main reasons. First, the huge column densities present in nuclear starbursts cause enormous extinction toward the central regions of (U)LIRGs at optical and infrared wavelengths, such that essentially no supernova would be detected at those wavelengths in the central few hundred par- 
secs of a (U)LIRG. Second, the enormous angular resolution needed to discern individual objects at the typical distances to (U)LIRGs ( $\gtrsim 50 \mathrm{Mpc}$ ) requires milliarcsecond angular resolution to resolve individual core-collapse supernovae (CCSNe) and/or supernova remnants (SNRs).

In this paper, we use high-angular radio observations from the literature, as well as data obtained by us, of three starburst galaxies in the local universe (M 82, Arp 299-A, and Arp 220) to analyze the radial distribution of CCSNe/SNRs in their central few hundred parsecs, which may have strong implications for the (co)-evolution of AGN and (nuclear) starbursts in galaxies, in general, and in (U)LIRGs in particular.

\section{The radial distribution of $\mathrm{SNe}$ in nuclear starbursts}

The radial distribution of $\mathrm{SNe}^{1}$ on galactic scales has been thoroughly studied (see, e.g., Bartunov et al. 1992; van den Bergh 1997; Anderson \& James 2009; Hakobyan et al. 2009). Optical observations have been very useful for this purpose, as dust extinction plays a minor role in the external regions of most galaxies. However, the dust-enshrouded environments of the nuclear regions in (U)LIRGs, as well as the need for milliarcsecond angular resolution to pinpoint individual $\mathrm{SNe}$, have prevented this kind of studies in the central regions of local (U)LIRGs. Fortunately, both issues - obscuration and (angular) resolution - can be overcome by means of Very Long Baseline Interferometry (VLBI) radio observations of the central regions of (U)LIRGs, since radio is dust-extinction free, and VLBI provides milliarsecond angular resolution.

\subsection{Methods}

We used VLBI observations to probe the radial distribution of $\mathrm{SNe}$ in the nuclear regions of Arp 299-A (31 objects) and Arp 220 (48 objects), as well as MERLIN, VLA, and VLBI observations toward M 82 (38 objects). For each galaxy, we fitted the surface density profile of the SNe to two different disk profiles: (i) an exponential disk, $\Sigma^{S N}=\Sigma_{0}^{S N} \exp \left(-\tilde{r} / \tilde{h}_{\mathrm{SN}}\right)$, and (ii) a disk with a power-law density profile with radius, $\Sigma^{S N}=$ $\Sigma_{0}^{S N}\left(r / r_{\text {out }}\right)^{-\gamma}$. The first case (exponential disk) assumes that the SN distribution follows the radial surface-brightness density in spiral disks (Freeman 1970), while the second one (power-law disk) was used to probe the profiles predicted by numerical simulations (Wada \& Norman 2002). The notation follows the one used in Hakobyan et al. (2009), where $\Sigma_{0}^{S N}$ is the surface density of $\mathrm{SN}$ at the center, $\tilde{r}$ is the normalized radius, and $r_{\text {out }}$ is the radius of the farthest SN analyzed, considered here to be the outer boundary of the putative nuclear disk. The main parameters that are obtained from those fits are the scale length, $\tilde{h}_{\mathrm{SN}}$, and the index of the power-law profile, $\gamma$, of the putative nuclear disks in the central regions of these galaxies.

Our approach consisted in model fitting the surface density profile of the radial distribution of SNe, by determining the number of sources in concentric rings around the center of the galaxy. For this purpose, we carried out a similar data analysis to the one described in Hakobyan et al. (2009, hereafter, H09), and we refer the reader to that paper for further details. In particular, we calculated the de-projected distance of each compact source to the center of the galaxy (the AGN, whenever its identification was possible) from the position angle of the galaxy major axis, and

\footnotetext{
${ }^{1}$ In this paper, the term $\mathrm{SNe}$ denotes both CCSNe and SNRs.
}

the inclination of the galactic disk. We obtained those parameter values from the HyperLeda database. We also followed H09, and measured radial distances in units of $R_{25}$, i.e., the isophotal radius at which the surface brightness was $25 \mathrm{mag} / \operatorname{arcsec}^{2}$ in the Johnson $B$ filter. We then derived the surface density profile by determining the number of sources in concentric rings around the nucleus, and fitting the data to both an exponential and a power-law disk, as described above.

Since the supernova samples for all our three galaxies are of relatively small size, resulting in some small size bins, the actual uncertainties in the fits could be larger than formally obtained from a single fit. To assess the robustness of our fitting procedure, we performed a series of Monte Carlo (MC) simulations. For each galaxy, we generated 10000 mock supernova samples, whose values were uniformly distributed within the uncertainty of each data point, and fitted them. In this way, we obtained a distribution of 10000 different fitting values for the scale-length parameter, $\tilde{h}_{\mathrm{SN}}$, and the power-law index, $\gamma$, for each galaxy. This approach allowed us to obtain more reliable values of those parameters, since no assumption was made about the Gaussianity of the distributions of both the scale length and power-law index, or of their errors. We then used the median value of the distribution of our MC values to characterize $\tilde{h}_{\mathrm{SN}}$ and $\gamma$, and set the uncertainty in these parameters at the $90 \%$ confidence level. To test our method, we reanalyzed the sample of H09 for 239 CCSNe within 216 host galaxies, and obtained a value of $\tilde{h}_{\mathrm{SN}}=0.29_{-0.01}^{+0.02}$, which is in excellent agreement with their estimate $^{2}$, and confirms the robustness of our analysis.

\subsection{Results}

We outline the results obtained for each individual galaxy. We refer the reader to the Appendices for details on the SN data used in our fitting analysis.

\subsubsection{Arp 299-A}

Arp 299-A ( $D=44.8 \mathrm{Mpc})$ is one of the two interacting galaxies of the LIRG Arp 299. The sample of SNe used for our study comes from both observations from the European VLBI Network (EVN) at $5.0 \mathrm{GHz}$, presented in Pérez-Torres et al. (2009) and in Bondi et al. (2012) (see also Appendix A), as well as from published data obtained with the Very Long Baseline Array (VLBA) at 2.3 and $8.4 \mathrm{GHz}$ by Ulvestad (2009). The existence and precise location of the low luminosity AGN in Arp 299-A was reported by Pérez-Torres et al. (2010), hence its position was used to calculate accurate de-projected distances for all SNe in the nuclear region of Arp 299-A. The sample has a total of 31 sources. However, although there are sources at distances as far as $\tilde{r}=11.4 \times 10^{-2}$, only one source is further than $\tilde{r}=6.6 \times 10^{-2}$, so the latter value of $\tilde{r}$ was used as the limiting radius for our study, giving a final number of 30 sources. The scale length obtained using the non-linear fit is $\tilde{h}_{\mathrm{SN}}=1.6 \times 10^{-3}$, with a standard deviation of $0.5 \times 10^{-3}$. After performing the MC analysis, we obtained a final value $\tilde{h}_{\mathrm{SN}}$ of $\left(1.9_{-0.8}^{+1.9}\right) \times 10^{-3}$ and $\Sigma_{0}^{S N}=\left(1.7_{-1.0}^{+2.8}\right) \times 10^{6}$.

${ }^{2} \mathrm{H} 09$ report $\tilde{h}_{\mathrm{SN}}=0.29 \pm 0.01$. We note that the explicit list of CCSNe is not given in $\mathrm{H} 09$, and therefore we could not reproduce exactly their sample. 


\subsection{2. $\operatorname{Arp} 220$}

Arp 220 is a merging system at $77 \mathrm{Mpc}$, and the closest and most well-studied ULIRG. High-angular resolution radio observations from its two nuclear regions were first obtained by Lonsdale et al. (2006) using global VLBI, who found a total of 49 radio supernovae in both the east (20) and west (29) nuclei. We used the position of the peak flux density from a delay-rate VLBI map as the estimated position for the center of each galactic nucleus (Parra et al. 2007). One of the compact sources in the Arp 220 east nucleus is at a much larger distance than the rest of them, which we did not use in our fit, thus limiting the normalized radii to $\tilde{r}=0.011$. Therefore, a total of $48 \mathrm{SNe}$ were considered in the fits, which we carried both separately, for each nucleus, and for the combined data set (Fig. A.3). Similarly, we performed MC simulations for each of the two nuclei, as well as for the combined sample In this way, we obtained a scale-length for the east and west nuclei of $\left(3.1_{-0.7}^{+2.0}\right) \times 10^{-3}$ and $\left(3.4_{-0.9}^{+1.5}\right) \times 10^{-3}$, respectively, and a combined scale-length for Arp 220 is $\left(3.3_{-0.4}^{+0.6}\right) \times 10^{-3}$. To quantify the effect of the uncertain position of the putative AGNs, we shifted them 100 times randomly around our nominal position, given by the peak flux density, by a maximum value of $\sigma_{(\mathrm{RA}, \mathrm{Dec})}=F W H M /(2 S / N)$, where FWHM is the full-width at half maximum, and $\mathrm{S} / \mathrm{N}$ is the signal-to-noise ratio, resulting in 4.2 mas for the east nucleus and 1.4 mas for the west one, and then fitted to the data. The maximum differences obtained were considered as a systematic error, which we added to our nominal values, such that the combined scale length became $\tilde{h}_{\mathrm{SN}}=\left(3.3_{-0.9}^{+0.7}\right) \times 10^{-3}$, with $\Sigma_{0}^{S N}=\left(7.6_{-2.1}^{+2.3}\right) \times 10^{5}$.

\subsubsection{Combination of Arp 299-A and Arp 220}

The normalized radii of Arp 299-A and Arp 220 (both east and west nuclei) are comparable. We can thus combine every source prior to the analysis to obtain an average scale-length parameter with smaller errors to help characterize both nuclear starbursts. The scale length obtained for the combined samples is $\tilde{h}_{\mathrm{SN}}=$ $\left(2.0_{-0.2}^{+0.3}\right) \times 10^{-3}$. The original non-linear fit is shown in Fig. 1 and our MC analysis results are shown in Fig. 2. After taking into account systematic errors due to the uncertainty in the precise location of the AGNs of the Arp 220 east and west nuclei, the final value for the scale length is $\tilde{h}_{\mathrm{SN}}=\left(2.0_{-0.4}^{+0.3}\right) \times 10^{-3}$ and $\Sigma_{0}^{S N}=\left(3.0_{-0.7}^{+0.9}\right) \times 10^{6}$.

\subsection{4. $M 82$}

At a distance of $3.2 \mathrm{Mpc}, \mathrm{M} 82$ is the prototypical starburst galaxy, which has been the target of many observations at highangular resolution. For our analysis, we combined sources catalogued as SNe (or SNR) detected by Wills et al. (1997), Allen \& Kronberg (1998), McDonald et al. (2002), Fenech et al. (2008), and Brunthaler et al. (2009). These comprise observations at several frequencies with MERLIN, VLA, and VLBA, including a total of 39 sources. Since no AGN has yet been discovered in M 82, we instead used the radio kinematic center of M 82 (Weliachew et al. 1984) as the position of its nucleus. The scale length obtained with the MC simulation is $\tilde{h}_{\mathrm{SN}}=$ $\left(2.8_{-0.3}^{+0.4}\right) \times 10^{-2}$. Although the radio kinematic center is likely to be very close, if not exactly coincident, with the putative nucleus of M 82, we nevertheless quantified the effect that an error in the position chosen as the nucleus of M 82 would have in the fits. In particular, we shifted our fiducial position for the

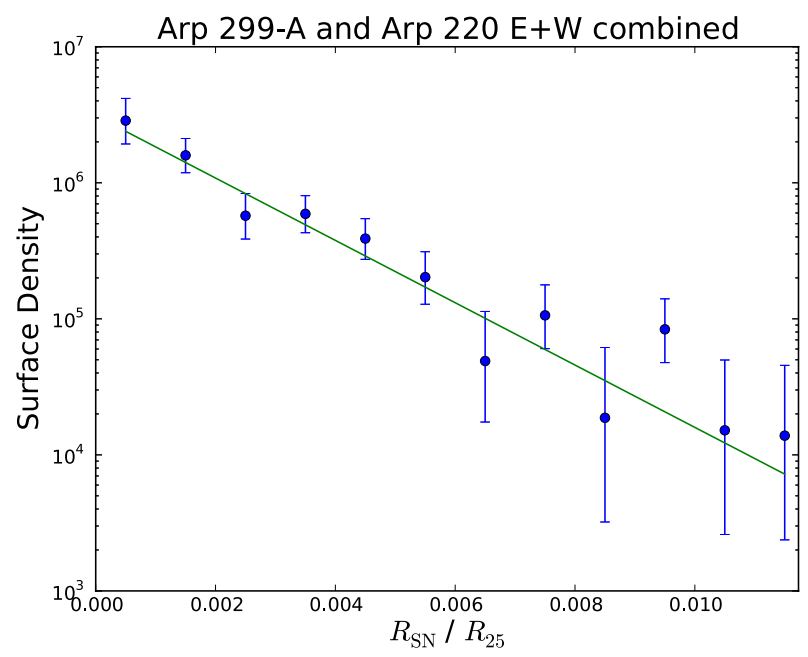

Fig. 1. Non-linear fit of the surface density profile of the combined sample of SNe from both Arp 299-A and Arp 220.

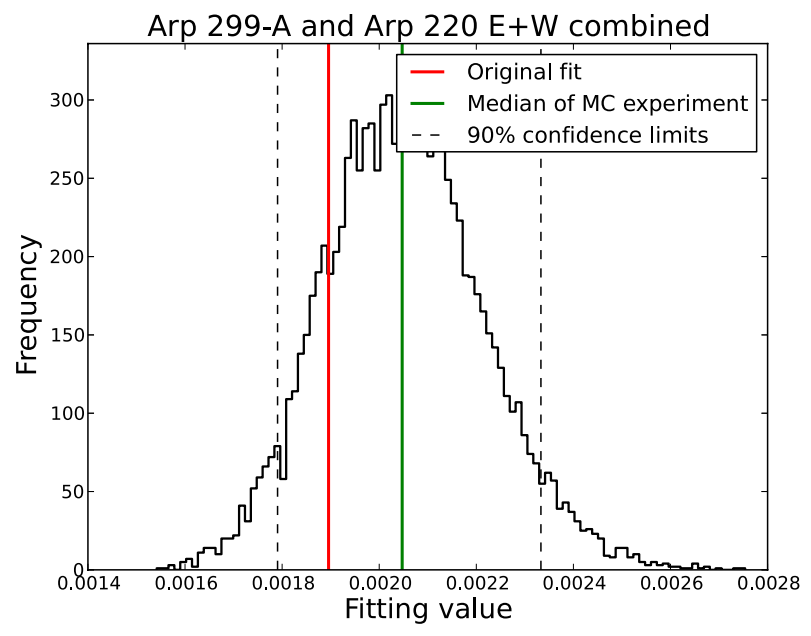

Fig. 2. MC histogram of $\tilde{h}_{\mathrm{SN}}$ for the combined sample of SNe from Arp 299-A and the two nuclei of Arp 220. The original non-linear fit is shown in red, and the median of the distribution created with the MC simulation is shown in green. The dashed lines correspond to the $90 \%$ confidence level interval.

nucleus by up to 20 parsec in several directions, and then fitted the data. The maximum differences were considered as a systematic error, which we added to our nominal value, thus yielding $\tilde{h}_{\mathrm{SN}}=\left(2.8_{-0.7}^{+0.9}\right) \times 10^{-2}$, and $\Sigma_{0}^{S N}=\left(8.3_{-2.3}^{+2.6}\right) \times 10^{3}$.

\section{Discussion and summary}

Our main results are summarized in Table 1 and Fig. 3, where we show the scale lengths, index of the power-law SN profile, and the radial distribution of $\mathrm{SNe}$ for the three galaxies discussed in this paper, as well as for the sample of spiral galaxies from H09.

We note that the scale lengths for the exponential disk fits to the radial distribution of $\mathrm{SNe}$ in the nuclear regions of the starburst galaxies, are at least an order of magnitude smaller that those obtained by H09 for the whole disks of spiral galaxies. In particular, $\tilde{h}_{\mathrm{SN}}$ is two orders of magnitude smaller in the case of Arp 299-A and Arp 220. Correspondingly, the physical sizes inferred for the scale lengths of the nuclear disks (Col. 3 in Table 1) are much smaller than for galactic disks: 20-30 pc for Arp 299-A or Arp 220, which is similar to the size derived for the nuclear starburst of the LIRG NGC 7469 


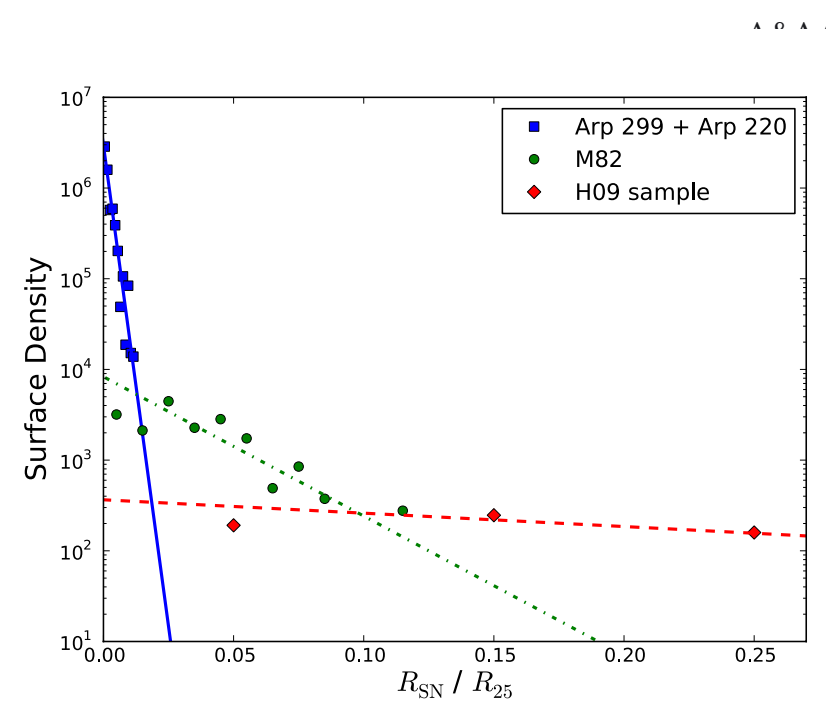

Fig. 3. Radial distribution of SNe for (nuclear) starbursts and spiral galaxies. The data correspond to the combined Arp 299-A + Arp 220 sample (blue squares), M 82 (green circles), and the H09 sample of spiral galaxies (red diamonds), and the fitted lines are for Arp 299-A + Arp 220 (solid line), M 82 (dotted-dashed line), and H09 (dashed line). For the sake of clarity, only the innermost part of the H09 sample is shown in the plot.

Table 1. Scale length parameters for the galaxies discussed in this paper.

\begin{tabular}{lccc}
\hline \hline Nucleus & $\tilde{h}_{\mathrm{SN}} / 10^{-3}$ & $h_{\mathrm{SN}}(\mathrm{pc})$ & $\gamma$ \\
\hline Arp 299-A & $1.9_{-0.8}^{+1.9}$ & $29.3_{-13.7}^{+29.6}$ & $1.1_{-0.2}^{+0.2}$ \\
Arp 220 East & $3.1_{-0.9}^{+2.0}$ & $22.2_{-6.2}^{+14.4}$ & $1.0_{-0.3}^{+0.2}$ \\
Arp 220 West & $3.4_{-1.5}^{+1.6}$ & $24.4_{-10.8}^{+11.2}$ & $0.8_{-0.2}^{+0.3}$ \\
Arp 220 E+W & $3.3_{-0.9}^{+0.7}$ & $23.4_{-6.6}^{+4.7}$ & $0.8_{-0.2}^{+0.1}$ \\
A299 + A220 & $2.0_{-0.4}^{+0.3}$ & $20.9_{-2.3}^{+2.6}$ & $0.9_{-0.1}^{+0.1}$ \\
M 82 & $\left(2.8_{-0.7}^{+0.9}\right) \times 10^{1}$ & $144.4_{-17.5}^{+21.5}$ & - \\
H09 sample & $\left(2.9_{-0.1}^{+0.2}\right) \times 10^{2}$ & - & - \\
\hline
\end{tabular}

Notes. All quoted uncertainties correspond to $90 \%$ confidence limit intervals, to which a systematic error was added in the cases of Arp 220 east, Arp 220 west, and M 82, owing to the uncertainty in the precise location of their centers.

(30-60 pc; Davies et al. 2004b), using CO interferometric observations, and $\sim 160 \mathrm{pc}$ for M 82 , which is also similar to the scale-length of the nuclear disk in the ULIRG/QSO Mrk 231 (Davies et al. 2004a).

We show in Fig. 3 the radial distribution of SNe in our three starburst galaxies and, for comparison, also for spiral galaxies (from the H09 sample), along with the fits to these data. We note the clear existence of three different regimes: one characterized by a very steep profile of the surface number density of SNe, which is typical of strong, nuclear starbursts (Arp 299A+Arp 220); a second, less steep profile, as indicated for the (circum)nuclear starburst in M 82; and a third, flatter profile, which is typical of very large disks, such as those in spiral galaxies (H09 sample). These results suggest that the surface density of SNe, hence of available gas to convert into stars, reaches a maximum in the vicinities of the SMBH in LIRGs and ULIRGs. We also show in Col. 4 of Table 1 the fits to a power-law disk profile, consistent with the fiducial value of $\gamma=1$ used by Wada \& Norman (2002) and Kawakatu \& Wada (2008) for the nuclear disk, and appears to yield further support to their modeling.
We have modeled for the first time the radial distribution of SNe in the nuclear starbursts of M 82, Arp 299-A, and Arp 220, and derived scale-length values for exponential disks, which are in the range between $\sim 20 \mathrm{pc}$ and $\sim 140 \mathrm{pc}$. We have also modeled these SNe distributions as power-law disk profiles, and found that they agree with state-of-the art numerical simulations of nuclear disks around SMBHs. We interpret our results as evidence of nuclear disks around the centers, i.e., AGNs, of starburstdominated galaxies and also support for evolutionary scenarios where a nuclear disk of size $\$ 100 \mathrm{pc}$ is formed in LIRGs and ULIRGs (Kawakatu \& Wada 2008). In particular, the scalelength obtained for the LIRG Arp 299-A and the LLAGN nature of its SMBH (Pérez-Torres et al. 2010), suggests that its nuclear disk is likely supported by gas pressure, such that the accretion onto the SMBH is smaller than for a turbulent-supported disk. It is very likely that this is also the case for the ULIRG Arp 220. Future deep VLBI observations of a significantly large sample of (U)LIRGs, which would result in the discovery of new SN factories, will be of much use for deriving statistically significant results on the size of nuclear disks, and thus setting useful constraints on (co)-evolutionary scenarios.

Acknowledgements. We are grateful to Rob Beswick and the anonymous referee for useful suggestions and comments. The authors acknowledge support by the Spanish MICINN through grant AYA2009-13036-CO2-01, partially funded by FEDER funds. This research has also been partially funded by the Autonomic Government of Andalusia under grants P08-TIC-4075 and TIC-126. We acknowledge the usage of the HyperLeda database (http://leda . univ-lyon1. $\mathrm{fr} /)$.

\section{References}

Allen, M. L., \& Kronberg, P. P. 1998, ApJ, 502, 218

Anderson, J. P., \& James, P. A. 2009, MNRAS, 399, 559

Bartunov, O. S., Makarova, I. N., \& Tsvetkov, D. I. 1992, A\&A, 264, 428

Bondi, M., Perez-Torres, M. A., Herrero-Illana, R., \& Alberdi, A. 2012, A\&A, 539, A134

Brunthaler, A., Menten, K. M., Reid, M. J., et al. 2009, A\&A, 499, L17

Davies, R. I., Tacconi, L. J., \& Genzel, R. 2004a, ApJ, 613, 781

Davies, R. I., Tacconi, L. J., \& Genzel, R. 2004b, ApJ, 602, 148

Di Matteo, P., Combes, F., Melchior, A.-L., \& Semelin, B. 2007, A\&A, 468, 61

Fenech, D. M., Muxlow, T. W. B., Beswick, R. J., Pedlar, A., \& Argo, M. K. 2008, MNRAS, 391, 1384

Freeman, K. C. 1970, ApJ, 160, 811

Hakobyan, A. A., Mamon, G. A., Petrosian, A. R., Kunth, D., \& Turatto, M. 2009, A\&A, 508, 1259

Kawakatu, N., \& Umemura, M. 2002, MNRAS, 329, 572

Kawakatu, N., \& Wada, K. 2008, ApJ, 681, 73

Lonsdale, C. J., Diamond, P. J., Thrall, H., Smith, H. E., \& Lonsdale, C. J. 2006, ApJ, 647, 185

McDonald, A. R., Muxlow, T. W. B., Wills, K. A., Pedlar, A., \& Beswick, R. J. 2002, MNRAS, 334, 912

Mihos, J. C., \& Hernquist, L. 1994, ApJ, 425, L13

Mihos, J. C., \& Hernquist, L. 1996, ApJ, 464, 641

Norman, C., \& Scoville, N. 1988, ApJ, 332, 124

Parra, R., Conway, J. E., Diamond, P. J., et al. 2007, ApJ, 659, 314

Pérez-Torres, M. A., Romero-Cañizales, C., Alberdi, A., \& Polatidis, A. 2009, A\&A, 507, L17

Pérez-Torres, M. A., Alberdi, A., Romero-Cañizales, C., \& Bondi, M. 2010, A\&A, 519, L5

Portegies Zwart, S. F., McMillan, S. L. W., \& Gieles, M. 2010, ARA\&A, 48, 431

Shlosman, I., Begelman, M. C., \& Frank, J. 1990, Nature, 345, 679

Simkin, S. M., Su, H. J., \& Schwarz, M. P. 1980, ApJ, 237, 404

Ulvestad, J. S. 2009, AJ, 138, 1529

Umemura, M. 2001, ApJ, 560, L29

Umemura, M., Fukue, J., \& Mineshige, S. 1997, ApJ, 479, L97

van den Bergh, S. 1997, AJ, 113, 197

Wada, K., \& Norman, C. A. 2002, ApJ, 566, L21

Weliachew, L., Fomalont, E. B., \& Greisen, E. W. 1984, A\&A, 137, 335

Wills, K. A., Pedlar, A., Muxlow, T. W. B., \& Wilkinson, P. N. 1997, MNRAS 291,517

Pages 5 to 7 are available in the electronic edition of the journal at http: //www . aanda. org 
R. Herrero-Illana et al.: Evidence of nuclear disks in starburst galaxies from their radial distribution of supernovae

Appendix A: Arp 299-A and Arp 220

Table A.1. Arp 299-A ${ }^{a}$ source list.

\begin{tabular}{|c|c|c|c|}
\hline Name & $\begin{array}{c}\Delta \alpha^{b} \\
(\mathrm{~J} 2000.0)\end{array}$ & $\begin{array}{c}\Delta \delta \\
(\mathrm{J} 2000.0)\end{array}$ & $\begin{array}{l}\text { Deprojected dist. } \\
\text { (mas) }\end{array}$ \\
\hline A3 & 33.5941 & 46.560 & 252.3 \\
\hline A 15 & 33.5991 & 46.637 & 190.9 \\
\hline $33.612+46.70^{c}$ & 33.6124 & 46.696 & 71.3 \\
\hline $33.615+46.67^{c}$ & 33.6154 & 46.667 & 47.9 \\
\hline A26 & 33.6163 & 46.652 & 55.6 \\
\hline $33.617+46.72^{c}$ & 33.6173 & 46.724 & 44.2 \\
\hline A18 & 33.6195 & 46.463 & 276.6 \\
\hline A19 & 33.6198 & 46.660 & 45.1 \\
\hline A6 & 33.6207 & 46.597 & 122.6 \\
\hline A0 & 33.6212 & 46.707 & 13.2 \\
\hline A2 & 33.6218 & 46.655 & 60.5 \\
\hline A27 & 33.6221 & 46.692 & 25.06 \\
\hline $33.624+46.77^{c}$ & 33.6241 & 46.771 & 81.9 \\
\hline $33.627+46.44^{c}$ & 33.6267 & 46.440 & 331.2 \\
\hline A28 & 33.6272 & 46.719 & 66.8 \\
\hline $33.628+46.62^{c}$ & 33.6280 & 46.623 & 135.8 \\
\hline $33.629+46.65^{c}$ & 33.6290 & 46.647 & 121.6 \\
\hline A7 & 33.6301 & 46.786 & 117.7 \\
\hline A 8 & 33.6305 & 46.401 & 394.6 \\
\hline A9 & 33.6307 & 46.620 & 159.4 \\
\hline A29 & 33.6344 & 46.915 & 249.6 \\
\hline A22 & 33.6355 & 46.677 & 160.6 \\
\hline A30 & 33.6362 & 46.863 & 209.1 \\
\hline A10 & 33.6392 & 46.551 & 291.6 \\
\hline A11 & 33.6403 & 46.582 & 272.9 \\
\hline A31 & 33.6441 & 46.629 & 271.1 \\
\hline A12 & 33.6495 & 46.590 & 347.3 \\
\hline A4 & 33.6500 & 46.537 & 394.0 \\
\hline A25 & 33.6523 & 46.701 & 311.8 \\
\hline A13 & 33.6531 & 46.733 & 309.6 \\
\hline A14 & 33.6825 & 46.570 & 666.2 \\
\hline
\end{tabular}

Notes. ${ }^{(a)}$ Arp 299-A has a major axis position angle of PA $=163.6^{\circ}$ and an inclination of its galactic disk of $i=52.4^{\circ} .{ }^{(b)}$ Coordinates are given with respect to $\alpha(\mathrm{J} 2000.0)=11: 28: 00.0000$ and $\delta(\mathrm{J} 2000.0)=$ 58:33:00.000 (see Bondi et al. 2012). ${ }^{(c)}$ Sources from Ulvestad (2009).

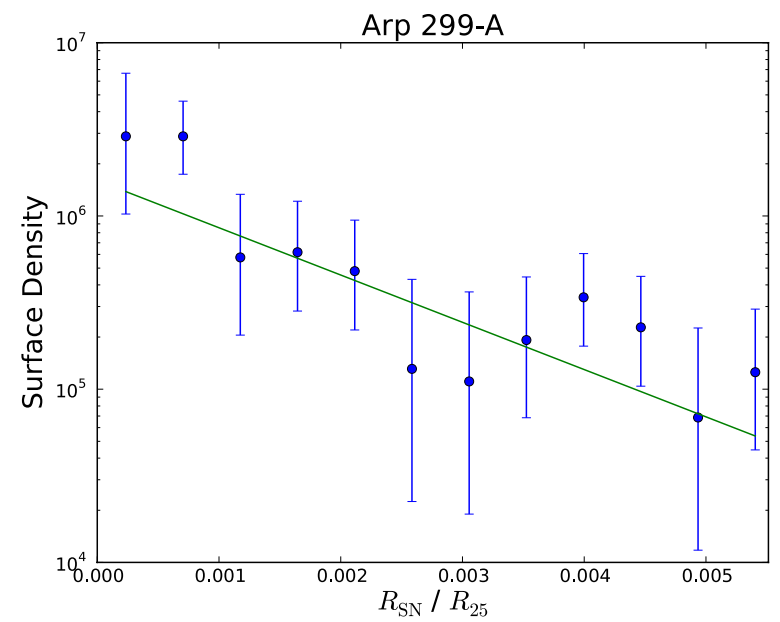

Fig. A.1. Surface density profile of the 30 inner SNe in Arp 299-A. The solid green line is a non-linear fit to an exponential disk with $\tilde{h}_{\mathrm{SN}}=$ $1.6 \times 10^{-3}$ and $\Sigma_{0}^{S N}=1.6 \times 10^{6}$.

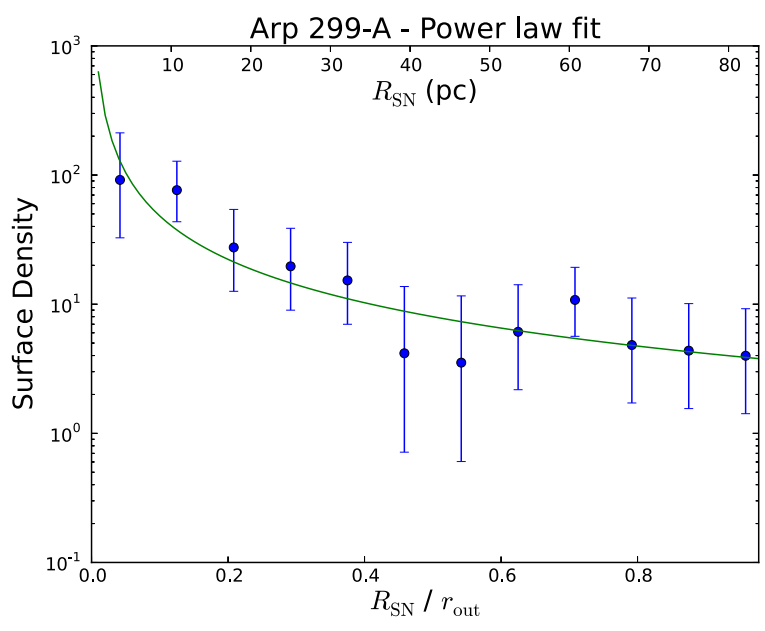

Fig. A.2. Surface density of $\mathrm{SNe}$ vs radii normalized to the furthest source, $r_{\text {out }}$, in Arp 299-A and non-linear fit to a power law with $\gamma=1.1$.
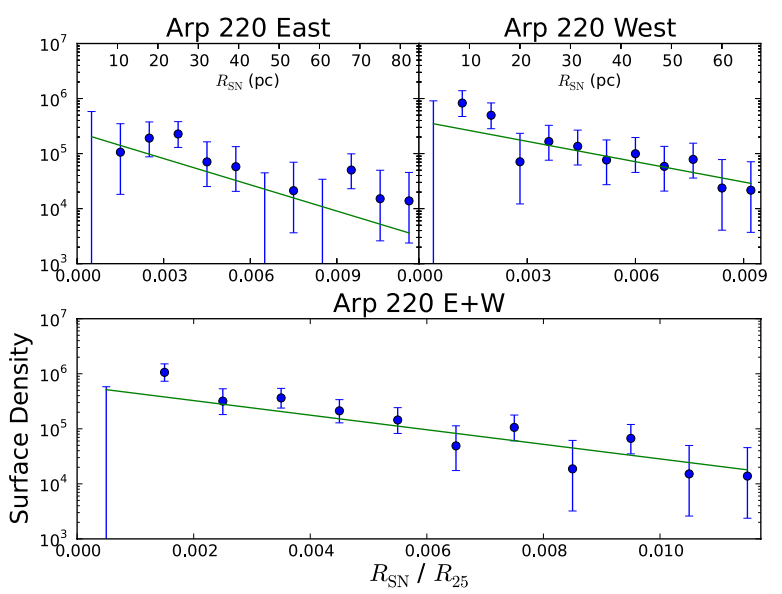

Fig. A.3. Non-linear fits to the surface density profile of the radial distribution of SNe in Arp 220. Top left: fit to the 19 inner sources in the eastern nucleus $\left(\tilde{h}_{\mathrm{SN}}=2.7 \times 10^{-3}\right)$. Top right: fit to the 29 sources in the western nucleus $\left(\tilde{h}_{\mathrm{SN}}=3.5 \times 10^{-3}\right)$. Bottom: combined fit for the $\mathrm{SNe}$ in the east and west nuclei (48 sources), yielding $\tilde{h}_{\mathrm{SN}}=3.3 \times 10^{-3}$ and $\Sigma_{0}^{S N}=6.0 \times 10^{5}$. We note that some of the points are in rings where no sources were detected. While their corresponding surface density is zero, their upper limits were used in the fit.

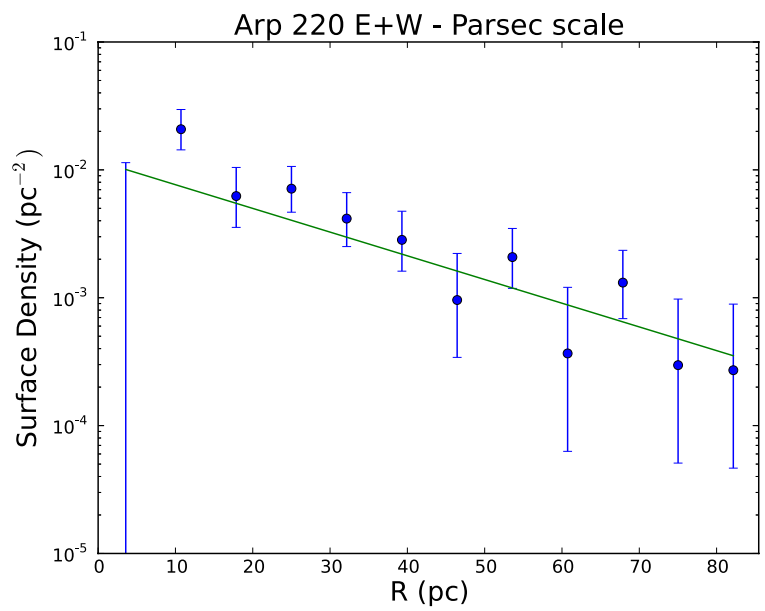

Fig. A.4. Surface density profile of the combined sources for the east and west nuclei of Arp 220, in parsecs. The non-linear fit yields a scale length of $h_{\mathrm{SN}}=23.4 \mathrm{pc}$, with $\Sigma_{0}^{S N}=1.2 \times 10^{-2} \mathrm{pc}^{-2}$. 
Table A.2. Arp $220^{a}$ source list.

\begin{tabular}{|c|c|c|c|}
\hline Name & $\begin{array}{c}\Delta \alpha^{b} \\
(\mathrm{~J} 2000.0)\end{array}$ & $\begin{array}{c}\Delta \delta \\
(\mathrm{J} 2000.0)\end{array}$ & $\begin{array}{l}\text { Deprojected dist. } \\
\text { (mas) }\end{array}$ \\
\hline W46 & 57.2066 & 11.459 & 310.4 \\
\hline W44 & 57.2082 & 11.548 & 254.8 \\
\hline W42 & 57.2123 & 11.482 & 215.6 \\
\hline W41 & 57.2161 & 11.405 & 264.9 \\
\hline W40 & 57.2162 & 11.478 & 167.4 \\
\hline W39 & 57.2171 & 11.484 & 150.3 \\
\hline W34 & 57.2195 & 11.492 & 112.1 \\
\hline W33 & 57.2200 & 11.492 & 106.0 \\
\hline W30 & 57.2211 & 11.398 & 235.7 \\
\hline W29 & 57.2212 & 11.445 & 157.1 \\
\hline W28 & 57.2212 & 11.541 & 82.8 \\
\hline W27 & 57.2219 & 11.387 & 251.9 \\
\hline W26 & 57.2223 & 11.435 & 164.4 \\
\hline W25 & 57.2223 & 11.500 & 69.0 \\
\hline W24 & 57.2224 & 11.514 & 58.1 \\
\hline W19 & 57.2240 & 11.499 & 48.9 \\
\hline W18 & 57.2240 & 11.546 & 62.8 \\
\hline W17 & 57.2241 & 11.520 & 34.3 \\
\hline W16 & 57.2244 & 11.531 & 39.7 \\
\hline W15 & 57.2253 & 11.483 & 62.4 \\
\hline W14 & 57.2270 & 11.573 & 107.1 \\
\hline W12 & 57.2295 & 11.524 & 47.1 \\
\hline W11 & 57.2300 & 11.503 & 50.0 \\
\hline W10 & 57.2306 & 11.502 & 57.8 \\
\hline W09 & 57.2318 & 11.401 & 209.3 \\
\hline W08 & 57.2360 & 11.431 & 186.6 \\
\hline W07 & 57.2362 & 11.547 & 154.4 \\
\hline W03 & 57.2387 & 11.401 & 248.7 \\
\hline E22 & 57.2758 & 11.277 & 169.3 \\
\hline E21 & 57.2789 & 11.293 & 127.4 \\
\hline E20 & 57.2821 & 11.185 & 189.2 \\
\hline E19 & 57.2840 & 11.151 & 235.3 \\
\hline E18 & 57.2843 & 11.226 & 104.8 \\
\hline E17 & 57.2850 & 11.193 & 156.5 \\
\hline E16 & 57.2848 & 11.472 & 361.0 \\
\hline E14 & 57.2868 & 11.297 & 46.8 \\
\hline E13 & 57.2878 & 11.308 & 66.3 \\
\hline E12 & 57.2885 & 11.212 & 109.5 \\
\hline E11 & 57.2910 & 11.325 & 111.7 \\
\hline E10 & 57.2914 & 11.332 & 126.4 \\
\hline E09 & 57.2915 & 11.477 & 384.8 \\
\hline E08 & 57.2931 & 11.328 & 132.9 \\
\hline E07 & 57.2943 & 11.279 & 90.5 \\
\hline E06 & 57.2946 & 11.264 & 91.2 \\
\hline E05 & 57.2980 & 11.419 & 319.7 \\
\hline E04 & 57.2986 & 11.408 & 306.3 \\
\hline E03 & 57.3073 & 11.333 & 305.8 \\
\hline E01 & 57.3103 & 11.577 & 676.1 \\
\hline
\end{tabular}

Notes. ${ }^{(a)}$ Arp 220 has an inclination angle of $57.0^{\circ}$, with a majoraxis position angle of $96.5^{\circ} .{ }^{(b)}$ Coordinates are given with respect to $\alpha(\mathrm{J} 2000.0)=15: 34: 00.0000$ and $\delta(\mathrm{J} 2000.0)=23: 30: 00.000$.

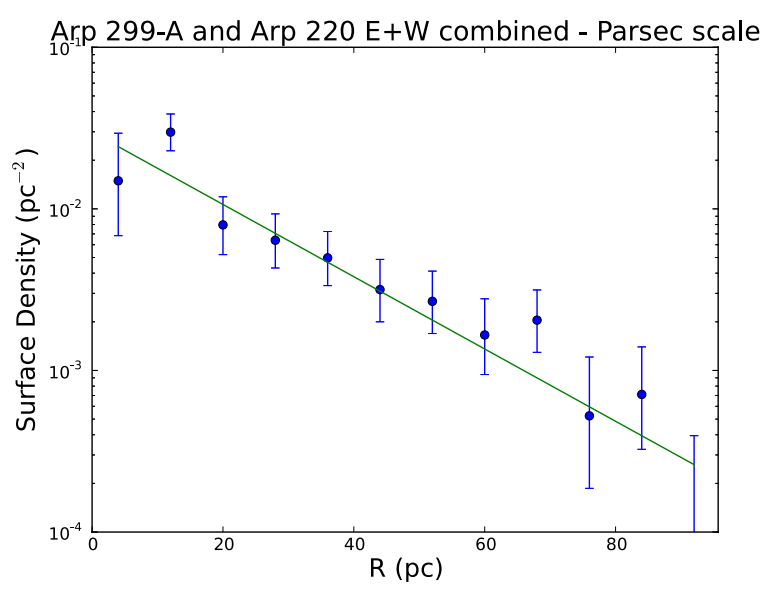

Fig. A.5. Surface density profile of the combined sources for Arp 299-A and Arp 220, in parsecs. The non-linear fit yields a scale-length of $h_{\mathrm{SN}}=$ $19.4 \mathrm{pc}$ and $\Sigma_{0}^{S N}=3.0 \times 10^{-2} \mathrm{pc}^{-2}$.
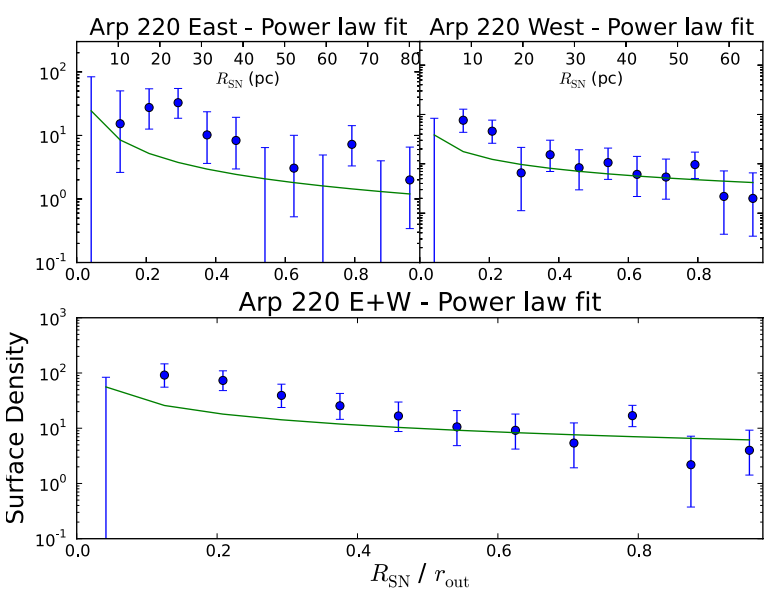

Fig. A.6. Non-linear power-law fits of the surface density of SNe vs. radius, normalized to the furthest source, $r_{\text {out }}$, in Arp 220. Top left: east nucleus, with $\gamma=1.0$. Top right: west nucleus, with $\gamma=0.7$. Bottom: combined analysis for the east and west nuclei (48 sources), yielding $\gamma=0.7$. Note that some of the points are in rings where no sources were detected. While their corresponding surface density is zero, their upper limits were used in the fit.

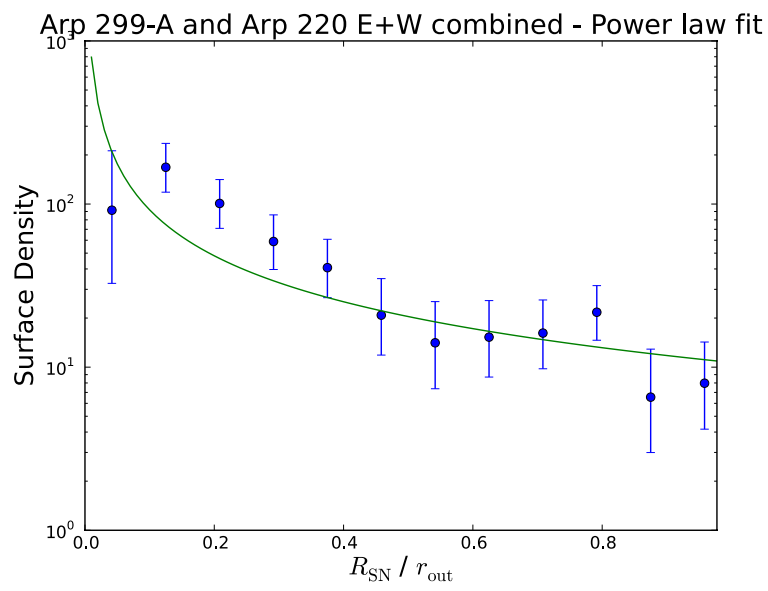

Fig. A.7. Surface density of $\mathrm{SNe}$ vs radii normalized to the furthest source, $r_{\text {out }}$, for the combined sample of SNe from Arp 299-A and the two nuclei of Arp 220. A non-linear fit to a power law with radius yields $\gamma=0.9$. 
R. Herrero-Illana et al.: Evidence of nuclear disks in starburst galaxies from their radial distribution of supernovae

\section{Appendix B: M 82}

Table B.1. M $82^{a}$ source list.

\begin{tabular}{|c|c|c|c|}
\hline Name $^{b}$ & $\begin{array}{c}\Delta \alpha^{\mathrm{c}} \\
(\mathrm{J} 2000.0)\end{array}$ & $\begin{array}{c}\Delta \delta \\
(\mathrm{J} 2000.0)\end{array}$ & $\begin{array}{l}\text { Deprojected dist. } \\
\text { (mas) }\end{array}$ \\
\hline $39.10+57.3$ & 47.87 & 43.74 & 37.03 \\
\hline $39.40+56.2$ & 48.16 & 42.53 & 29.20 \\
\hline $39.64+53.3$ & 48.41 & 39.68 & 20.00 \\
\hline $39.77+56.9$ & 48.54 & 43.36 & 27.84 \\
\hline $40.32+55.2$ & 49.07 & 41.53 & 16.98 \\
\hline $40.61+56.3$ & 49.32 & 42.19 & 15.93 \\
\hline $40.68+55.1$ & 49.42 & 41.42 & 14.13 \\
\hline $41.30+59.6$ & 50.05 & 45.92 & 20.40 \\
\hline $41.95+57.5$ & 50.70 & 44.00 & 6.94 \\
\hline $42.61+60.7$ & 51.35 & 46.89 & 9.35 \\
\hline $42.66+51.6$ & 51.38 & 47.76 & 13.21 \\
\hline $42.67+55.6$ & 51.37 & 41.78 & 17.44 \\
\hline $42.67+56.3$ & 51.40 & 42.65 & 13.42 \\
\hline $42.80+61.2$ & 51.54 & 47.53 & 10.25 \\
\hline $43.18+58.2$ & 51.91 & 44.58 & 8.70 \\
\hline $43.31+59.2$ & 52.03 & 45.42 & 5.75 \\
\hline $43.40+62.6$ & 52.11 & 48.74 & 10.22 \\
\hline $43.55+60.0$ & 52.27 & 46.31 & 4.22 \\
\hline $43.72+62.6$ & 52.43 & 48.79 & 7.63 \\
\hline $43.82+62.8$ & 52.54 & 48.98 & 7.71 \\
\hline $44.01+59.6$ & 52.72 & 45.77 & 12.03 \\
\hline $44.08+63.1$ & 52.75 & 49.46 & 8.50 \\
\hline $44.28+59.3$ & 52.99 & 45.53 & 16.33 \\
\hline $44.34+57.8$ & 53.05 & 43.94 & 24.65 \\
\hline $44.40+61.8$ & 53.13 & 47.87 & 8.35 \\
\hline $44.51+58.2$ & 53.22 & 44.36 & 24.59 \\
\hline $44.89+61.2$ & 53.61 & 47.35 & 15.69 \\
\hline $45.17+61.2$ & 53.88 & 47.41 & 18.61 \\
\hline $45.24+65.2$ & 53.96 & 51.36 & 12.15 \\
\hline $45.42+67.4$ & 54.13 & 53.53 & 18.13 \\
\hline $45.52+64.7$ & 54.22 & 50.94 & 12.94 \\
\hline $45.75+65.3$ & 54.44 & 51.43 & 14.17 \\
\hline $45.79+64.0$ & 54.50 & 50.22 & 16.24 \\
\hline $45.89+63.8$ & 54.60 & 49.98 & 17.85 \\
\hline $46.52+63.9$ & 55.21 & 50.05 & 24.19 \\
\hline $46.56+73.8$ & 55.26 & 59.90 & 38.39 \\
\hline $46.70+67.1$ & 55.41 & 53.16 & 19.71 \\
\hline $47.37+68.0$ & 56.10 & 54.00 & 24.01 \\
\hline SN2008iz & 51.55 & 45.79 & 2.41 \\
\hline
\end{tabular}

Notes. ${ }^{a} \mathrm{M} 82$ has a major position angle of $67.5^{\circ}$ and an inclination of $79.4^{\circ} .{ }^{b}$ Names from the literature are derived from B1950 coordinates. ${ }^{c}$ Coordinates are given with respect to $\alpha(\mathrm{J} 2000.0)=09: 55: 00.00$ and $\delta(\mathrm{J} 2000.0)=69: 40: 00.00$.

\section{Appendix C: Nature of the radio sources}

The sources used in this paper are classified generically as supernovae ( $\mathrm{SNe})$, by which we mean core collapse supernovae (CCSNe) and SNRs. In the case of M 82, the closest of the three galaxies discussed here, we used the source list in McDonald et al. (2002) and Fenech et al. (2008), where the authors already discriminate $\mathrm{SNe}$ from $\mathrm{HII}$ regions based on their spectral shape, brightness temperature, and observed shell structure. In the cases of the more distant galaxies, Arp 299 and Arp 220, we used available spectral information from the literature, and especially, the brightness temperatures inferred from VLBI observations, which for all of the sources correspond to non-thermal radio emitters (e.g., Pérez-Torres et al. 2009, for Arp 299A; Parra et al. 2007, for Arp 220).

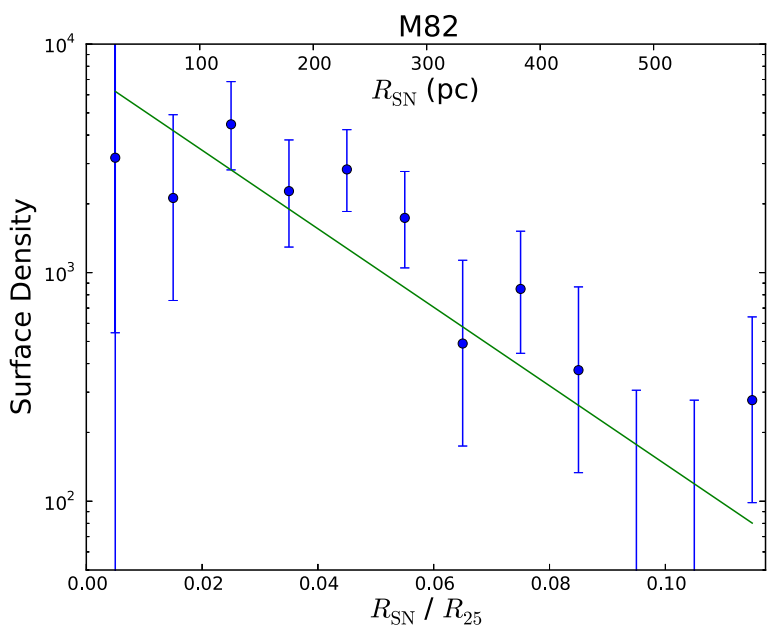

Fig. B.1. Surface density profile for the radial distribution of $\mathrm{SNe}$ in M 82 (39 objects). The solid green line is a non-linear fit to an exponential disk with $\tilde{h}_{\mathrm{SN}}=2.5 \times 10^{-2}$ and $\Sigma_{0}^{S N}=7.5 \times 10^{3}$.

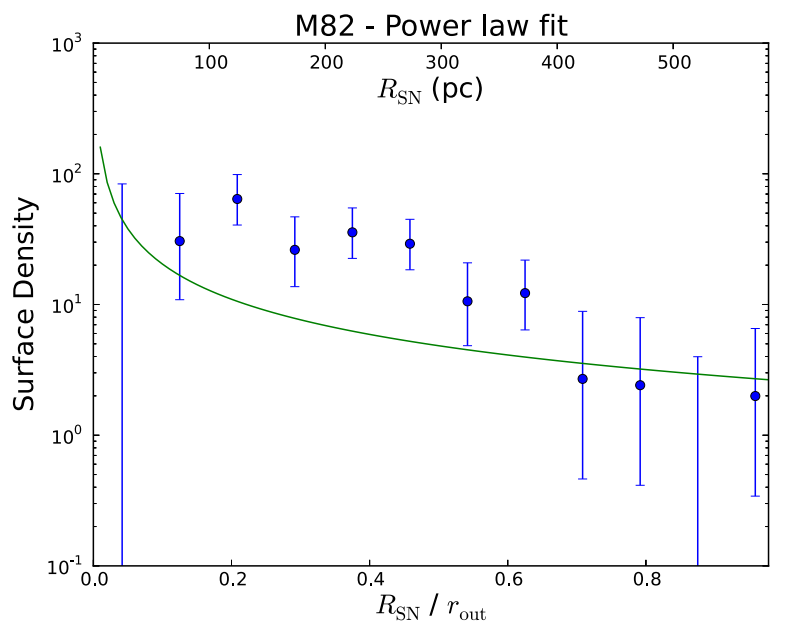

Fig. B.2. Surface density of SNe vs. radii normalized to the furthest source, $r_{\text {out }}$ and non-linear fit to a power law (nuclear) disk for M 82 . The poor goodness of the fit does not seem to be consistent with a nuclear disk whose SN surface density follows a power law in the central few hundred pc.

Single or multiple ultra compact HII (UC HII) regions can be ruled out because their ionizing radiation is normally dominated by a single $\mathrm{O}$ star, whose maximum free-free thermal radio luminosity is several orders of magnitude below the radio luminosity from any of the objects detected in either Arp 299A or Arp 220. The expected thermal radio emission from super star clusters (SSCs), which can host up to a few $10^{5}$ stars, similarly cannot be responsible for the observed emission. At the distances of Arp 299 and Arp 220, the VLBI beam sizes correspond to $\sim 1.5 \mathrm{pc}$, implying radial sizes of at most about $0.8 \mathrm{pc}$. Young massive stellar clusters of this size have a mass of at most $5 \times 10^{5} M_{\odot}$ (e.g. Portegies Zwart et al. 2010). Assuming a Kroupa initial mass function with $\alpha=-1.0$ between 0.1 and $0.5 M_{\odot}$, and $\alpha=-2.35$ between 0.5 and $100 M_{\odot}$, the number of O6 and earlier type stars is 142 (out of a total of $10^{5}$ stars), hence the maximum attainable thermal radio luminosity would be $\sim 2 \times 10^{25} \mathrm{erg} / \mathrm{s} / \mathrm{Hz}$, which is at least an order of magnitude lower than observed in Arp 299-A or Arp 220. 